\title{
Implementation of a standardized pregnancy screening process to address gender disparities in radiology turn-around-time and ED length of stay
}

\author{
Dana E. Loke ${ }^{1}$ D $\cdot$ Andra M. Farcas ${ }^{1}$ - Justine S. Ko ${ }^{1} \cdot$ Laurie M. Aluce $^{1}$ - Valerie R. McDonald ${ }^{1} \cdot$ Nahzinine Shakeri $^{1}$. \\ Abra L. Fant ${ }^{1}$
}

Received: 17 May 2021 / Accepted: 27 October 2021 / Published online: 11 January 2022

( ) The Author(s), under exclusive licence to Canadian Association of Emergency Physicians (CAEP)/ Association Canadienne de Médecine d'Urgence (ACMU) 2022

\begin{abstract}
Objective The objective of this initiative was to quantify and intervene upon suspected gender disparities in CT turn-aroundtime and emergency department (ED) length of stay.

Methods This was a single-site before-after quality improvement initiative including patients aged 12-50 who underwent CT chest and/or abdomen/pelvis. The intervention included protocolization of the pregnancy screening process in triage. Primary outcomes included the difference between women of childbearing age and similarly aged men in regards to CT turn-around-time and ED length of stay. Pre- and post-intervention data were analyzed, including an "intensive intervention period" subanalysis.

Results CT turn-around-time for women of childbearing age was 19 min longer than for similarly aged men at baseline and did not change significantly post-intervention. ED length of stay was 27 min longer for women of childbearing age compared to similarly aged men at baseline and 7 min longer post-intervention, although this was still a significant difference. During the intensive intervention period, CT turn-around-time for women of childbearing age was 15 min longer than similarly aged men but the difference in ED length of stay of 10 min was no longer significant.

Conclusions There is gender disparity in CT turn-around-time and ED length of stay in our ED, highlighting an important area for improvement to promote equitable care. A quality improvement initiative that aimed to protocolize pregnancy testing in triage did not show sustainable improvement in these outcomes but did result in increased pregnancy testing.
\end{abstract}

Keywords Gender disparities $\cdot$ Patient safety $\cdot$ Radiology turn-around-time $\cdot$ Length of stay $\cdot$ Women of childbearing age

Dana E. Loke

dana.loke@northwestern.edu

Andra M. Farcas

andra.malina.farcas@gmail.com

Justine S. Ko

justine.s.ko@gmail.com

Laurie M. Aluce

laurie.nosbusch@northwestern.edu

Valerie R. McDonald

valerie.mcdonald@nm.org

Nahzinine Shakeri

nahzinine.shakeri@northwestern.edu

Abra L. Fant

abra.berg@ northwestern.edu

1 Department of Emergency Medicine, Northwestern

University Feinberg School of Medicine, 211 E Ontario St,

Suite 200, Chicago, IL 60611, USA 


\section{Résumé}

Objectif L'objectif de cette initiative était de quantifier et d'intervenir sur les disparités présumées entre les sexes dans le délai d'exécution du scanner et la durée du séjour au service des urgences.

Méthodes Il s'agissait d'une initiative d'amélioration de la qualité à un seul site avant-après, incluant les patients âgés de 12 à 50 ans qui ont subi une TDM thoracique et/ou abdominale/bassin. L'intervention comprenait la protocolisation du processus de dépistage de la grossesse lors du triage. Les résultats primaires comprenaient la différence entre les femmes en âge de procréer et les hommes du même âge en ce qui concerne le délai d'exécution du scanner et la durée du séjour aux urgences. Les données avant et après l'intervention ont été analysées, y compris une sous-analyse de la "période d'intervention intensive". Résultats Le temps d'exécution de la TDM chez les femmes en âge de procréer était 19 minutes de plus que chez les hommes de même âge au départ et n'a pas changé de façon significative après l'intervention. La durée de séjour aux urgences était de 27 minutes de plus pour les femmes en âge de procréer par rapport aux hommes du même âge au départ et de 7 minutes de plus après l'intervention, bien que cette différence reste significative. Au cours de la période d'intervention intensive, le temps de passage au scanner des femmes en âge de procréer était de 15 minutes plus long que celui des hommes du même âge, mais la différence de 10 minutes dans la durée de séjour aux urgences n'était plus significative.

Conclusions Il existe une disparité entre les sexes en ce qui concerne le délai d'exécution du scanner et la durée de séjour dans nos urgences, ce qui met en évidence un domaine important à améliorer pour promouvoir des soins équitables. Une initiative d'amélioration de la qualité visant à protocoliser le test de grossesse au triage n'a pas montré d'amélioration durable de ces résultats mais a entraîné une augmentation des tests de grossesse.

\section{Clinician's capsule}

\section{What is known about the topic?}

Gender disparities exist in all areas of medicine and can lead to harmful and inequitable delays in care.

\section{What did this study ask?}

Can a standardized urine pregnancy screening process address suspected gender disparities in CT turnaround-time and length of stay in our ED?

\section{What did this study find?}

There are gender disparities in CT turn-around-time and ED length of stay in our ED, which were not significantly changed post-intervention.

\section{Why does this study matter to clinicians?}

A sustainable intervention that expedites pregnancy screening, such as rapid serum pregnancy testing, is needed.

\section{Introduction}

Confirming pregnancy status is pivotal to providing safe care to women of childbearing age in the emergency department (ED). Numerous studies have shown the teratogenic effects of radiation on a fetus, and many organizations, including the American College of Radiology, have recommended pregnancy screening prior to Computed Tomography (CT) imaging in areas such as the abdomen and pelvis [1,2]. As a result, unknown pregnancy status can cause diagnostic and treatment delays. These delays can lead to adverse downstream effects, such as longer ED length of stay and may be a cause of gender disparity in care. Delays within the ED have been shown to extend beyond the department with increased hospital length of stay and poor patient satisfaction $[3,4]$. ED crowding can lead to an increased risk of in-hospital mortality and longer times to treatment, while imaging delays themselves predict longer hospital length of stay for emergency medical admissions $[5,6]$.

Providers within our ED have observed delays in CT imaging for women of childbearing age, but there has been no investigation to quantify these delays. We suspected these delays may be in part related to pregnancy screening, which, per institutional protocol, is required for women of childbearing age undergoing $\mathrm{CT}$ chest and/or abdomen/pelvis unless they meet certain exemption criteria (e.g., hysterectomy, trauma, need for emergent imaging, patient declines testing).

It is the standard of care in our institution to obtain a point-of-care urine pregnancy test, which has an accuracy of $>99 \%$ [7]. While the use of this test is a long-standing institutional practice and accepted clinical practice, the process of obtaining the test is not standardized $[8,9]$. Our primary aim was to quantify and eliminate disparities in CT turn-around-time and ED length of stay between men and women aged $12-50$ through a quality improvement initiative to protocolize pregnancy screening for all women of childbearing age. Additionally, we sought to increase the percentage of women of childbearing age with pregnancy screening performed in the ED as a secondary aim. We describe our quality improvement initiative using the SQUIRE V.2.0 framework [10]. 


\section{Methods}

\section{Study design and time period}

A single-site before-after quality improvement initiative was conducted to quantify gender disparities and implement a protocolized pregnancy screening process to reduce disparities in CT turn-around-time and ED length of stay in women of childbearing age compared to similarly aged men. The intervention involved protocolization of the pregnancy screening process in triage using the institution's existing point-of-care urine pregnancy test. Pre-intervention data were collected March 2018-January 2019. Post-intervention data were collected February-July 2019. The project was deemed non-human subjects research by the Institutional Review Board.

\section{Study setting, population, and selection of participants}

Data were gathered in the ED of a large, urban, academic Level I Trauma centre that delivers care to over 100,000 primarily adult patients annually and has 2 CT scanners available at all times. The baseline process for pregnancy screening prior to CT imaging is represented in the process map in Fig. 1. Before the intervention period, triage nurses performed and documented point-of-care urine pregnancy testing ad hoc with assistance from ED Assistants, but this was not mandatory or protocolized. For all other women of childbearing age, pregnancy testing was performed after clinician evaluation. In cases where a pregnancy test was not documented at the time the CT technician arrived for transport, the patient was skipped until a pregnancy test was documented.

All patients aged 12-50 years who underwent CT chest and/or abdomen/pelvis were included in the primary aim analysis, as women within this age range are required to have a negative pregnancy test prior to these scans unless exemption criteria are met. Patients were excluded if deemed Left Without Being Seen or ED Dismissed-Never Arrived, if CT order was placed after CT completion or after the patient departed the ED, or if the CT was completed after the patient departed the ED. In addition, a second population consisting of all women aged 12-50 years presenting to the ED was used for secondary aim analysis of overall pregnancy screening rates.

\section{Intervention}

From February to July 2019, a staff-centred intervention involving protocolization of urine pregnancy testing in ED triage was implemented. In the primary quality improvement cycle, labeled the "intensive intervention" period, bi-daily staff education sessions continued for 8 weeks at team huddles. The goal of performing pregnancy screening earlier in the patient-care timeline was explained. Triage staff were asked to provide a specimen cup to all women of childbearing age, perform point-of-care urine pregnancy testing, and document the result. Signs reinforcing the process were placed in highly visible areas including break rooms, triage, and testing stations. Additional intervention components included optimizing stocking of supplies and

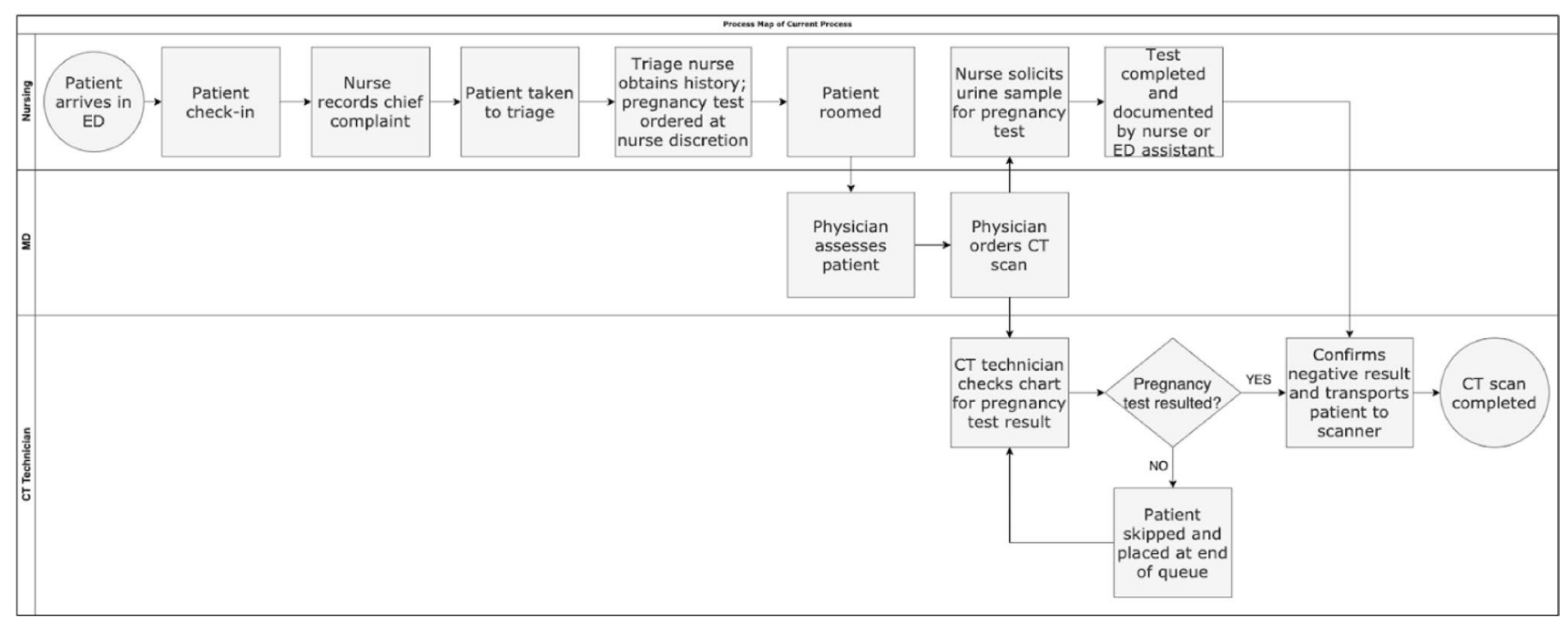

Fig. 1 Process map of baseline process for pregnancy screening prior to CT imaging 
posting signs in patient bathrooms encouraging women of childbearing age to provide a urine sample. The primary quality improvement cycle was extended after the initial 8 weeks to assess for intervention sustainability. During the remaining intervention period, the staff education sessions were discontinued. A repeat analysis was performed on the extended quality improvement cycle.

\section{Outcome measurements}

Primary outcome measures included CT turn-around-time, defined as time from CT order to CT acquisition, and ED length of stay, defined as time from ED arrival to departure, and the difference between women of childbearing age and similarly aged men for each of these measures. The secondary outcome measure was the percentage of women of childbearing age with pregnancy screening performed in the ED. Our process measure was the percentage of pregnancy tests ordered from triage. Creatinine result time, defined as time from creatinine order placement to result, was analyzed as a potential confounder, as this is the only other test result required before CTs with intravenous contrast.

\section{Data analysis}

Data were collected from the institution's enterprise data warehouse. Descriptive statistics were analyzed using JASP software to find gender-specific means for age, CT turnaround-time, ED length of stay, and creatinine result time. Student's $T$-tests were performed with $p<0.05$ to compare women of childbearing age to similarly aged men in each data category, as well as pre- to post-intervention within each gender group. When Levene's test was significant, a Mann U Whitney $T$-test was run with $p<0.05$. Data were further organized into run charts for delta between women of childbearing age and similarly aged men in CT turn-aroundtime and ED length of stay. A subset of the post-intervention data (February 2019-March 2019) was designated for subanalysis as the time immediately after implementation of the intervention when educational sessions were ongoing for the primary quality improvement. This subset has been designated the "intensive intervention" period.

Although the intervention focused on protocolizing pointof-care urine pregnancy testing, serum quantitative and lab-run urine testing were also included as valid pregnancy screening options. A chi-square test was used to compare pre- and post-intervention values with $p<0.05$. The percentage of pregnancy tests performed in the ED and ordered from triage for the three time periods were analyzed and a chi-square test was used to compare pre- and post-intervention values with $p<0.05$.

\section{Results}

Figure 2 demonstrates patient selection. Overall, 5215 patients were included in the pre-intervention period and 2907 in the post-intervention period.

A comparison of the primary outcomes during the preintervention, intensive intervention, and post-intervention periods is shown in Table 1. Women of childbearing age had longer CT turn-around-time compared to similarly aged men in all three periods. The disparity between the two groups is shown as a run chart in Fig. 3. ED length of stay was significantly longer for women of childbearing age in the pre-intervention and post-intervention period, but there was no significant difference between the groups in the intensive intervention period. The delta between the groups is shown as a run chart in Fig. 4. There was no difference in creatinine result time between the two groups (Table 1).

Data for pregnancy testing for all women of childbearing age who presented to the ED and for those who underwent $\mathrm{CT}$ chest and/or abdomen/pelvis are shown in Table 2. The percentage of testing in all women of childbearing age significantly increased with the intervention. The percentage of testing ordered from triage significantly increased during the intensive intervention period but decreased post-intervention. Pregnancy testing prior to CT scan remained stable.

Of the pregnancy tests performed, the types were similar throughout each period (88-90\% point-of-care urine tests, $7-8 \%$ serum quantitative tests, and 1-2\% lab-run urine tests).

\section{Discussion}

Pre-intervention data demonstrate statistically significant gender disparities in CT turn-around-time and ED length of stay. We suspected that these disparities are related to delays in pregnancy testing, as this is the only additional requirement prior to $\mathrm{CT}$ imaging for women of childbearing age compared to similarly aged men. While we did not achieve our primary aim of eliminating gender disparities in CT turn-around-time and ED length of stay, there was a decrease in disparity between the two groups, suggesting that our intervention may have had some effect on these endpoints (Table 1, Figs. 3 and 4). However, while some of our results show improvement and even statistical significance, not all of the results are clinically meaningful or solely attributable to our intervention. This is particularly notable when considering the decrease in ED length of stay, which can be impacted by other confounding variables (Fig. 4). Our intervention did result in statistically significant improvements in our secondary aim of pregnancy testing for all women of childbearing age presenting to the ED. During the intensive intervention period, testing for all women of 


\section{Pre-intervention}

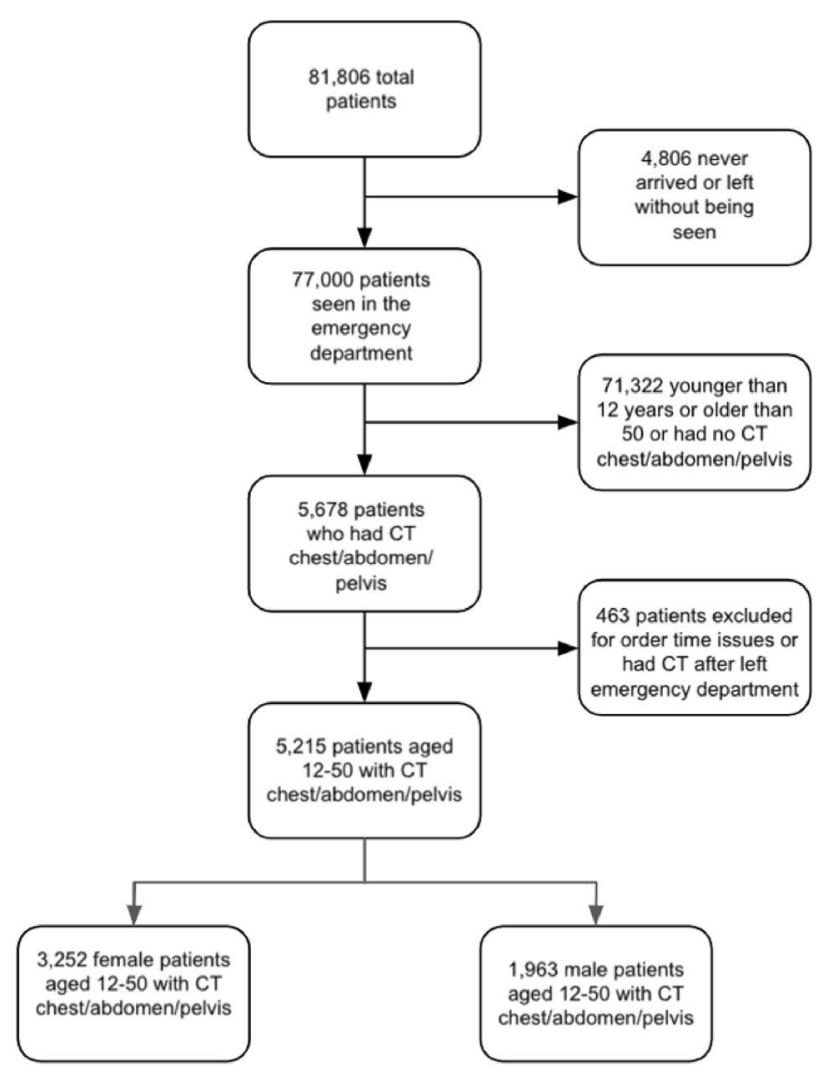

\section{Post-intervention}

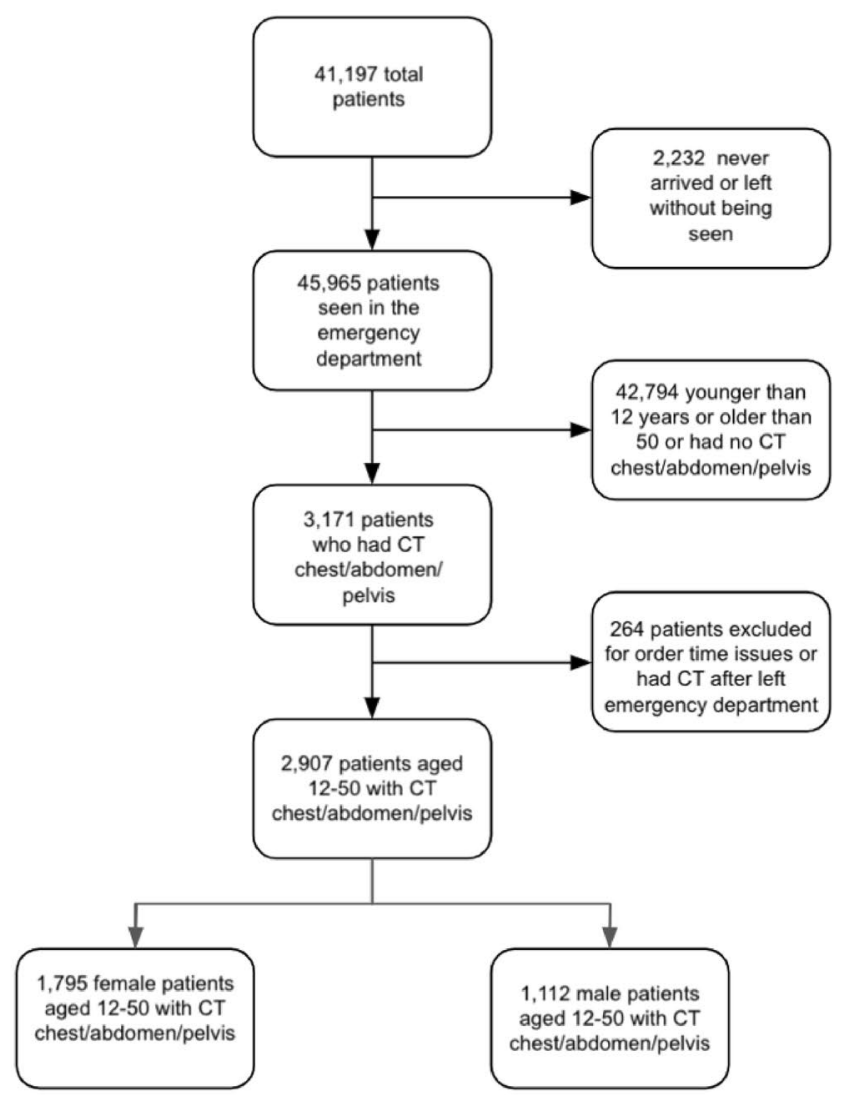

Fig. 2 Patient selection flow charts for pre-intervention and post-intervention periods

Table 1 Comparison between women of childbearing age and similarly aged men of baseline characteristics, CT turn-around-time, Emergency Department Length-of-Stay, and Creatinine Result Time over three time periods (pre-intervention, intensive intervention, and post-intervention)

\begin{tabular}{|c|c|c|c|c|c|c|c|c|c|}
\hline & \multicolumn{3}{|c|}{ Pre-intervention } & \multicolumn{3}{|c|}{ Intensive intervention } & \multicolumn{3}{|c|}{ Post-intervention } \\
\hline & Female & Male & $P$ & Female & Male & $P$ & Female & Male & $P$ \\
\hline$N$ & 3252 & 1963 & & 625 & 340 & & 1795 & 1112 & \\
\hline Age (years) & 34.8 & 35.9 & $<0.001$ & 34.8 & 35.7 & 0.145 & 34.8 & 36.1 & $<0.001$ \\
\hline $\begin{array}{l}\text { CT turn-around-time in minutes } \\
\text { (change from pre) }\end{array}$ & 108.6 & 89.9 & $<0.001$ & $109.3(+0.7)$ & $94.6(+4.7)$ & $<0.001$ & $109.5(+0.9)$ & $92.6(+2.7)$ & $<0.001$ \\
\hline $\begin{array}{l}\text { ED length of stay in minutes } \\
\text { (change from pre) }\end{array}$ & 525.4 & 498.5 & 0.01 & $538.4(+13)$ & $528.4(+29.9)$ & 0.716 & $532.6(+7.2)$ & $526.0(+27.5)$ & $<0.001$ \\
\hline $\begin{array}{l}\text { Creatinine result time in minutes } \\
\text { (change from pre) }\end{array}$ & 75.5 & 71.0 & 0.436 & $70.8(-4.7)$ & $67.5(-3.5)$ & 0.209 & $68.4\left(-7.1^{*}\right)$ & $67.8(-3.2)$ & 0.683 \\
\hline
\end{tabular}

*Denotes statistically significant change from pre-intervention

childbearing age increased by $7 \%$, but this improvement was not sustained throughout the entire post-intervention period. Therefore, our intervention did not result in clinically meaningful improvement in testing. Although we had intended for our intervention to result in a more robust increase in testing, we recognize it is unrealistic to expect $100 \%$ pregnancy screening. For many women of childbearing age who present to the ED, pregnancy testing is not indicated and may delay their care or add unnecessary cost. However, the fact that there was an overall increase in pregnancy testing 
Fig. 3 Run chart of the delta for average CT turn-around-time for women of childbearing age compared to similarly aged men by month. Delta indicates the difference between the values for women of childbearing age and similarly age men. The vertical line represents the start of the intervention

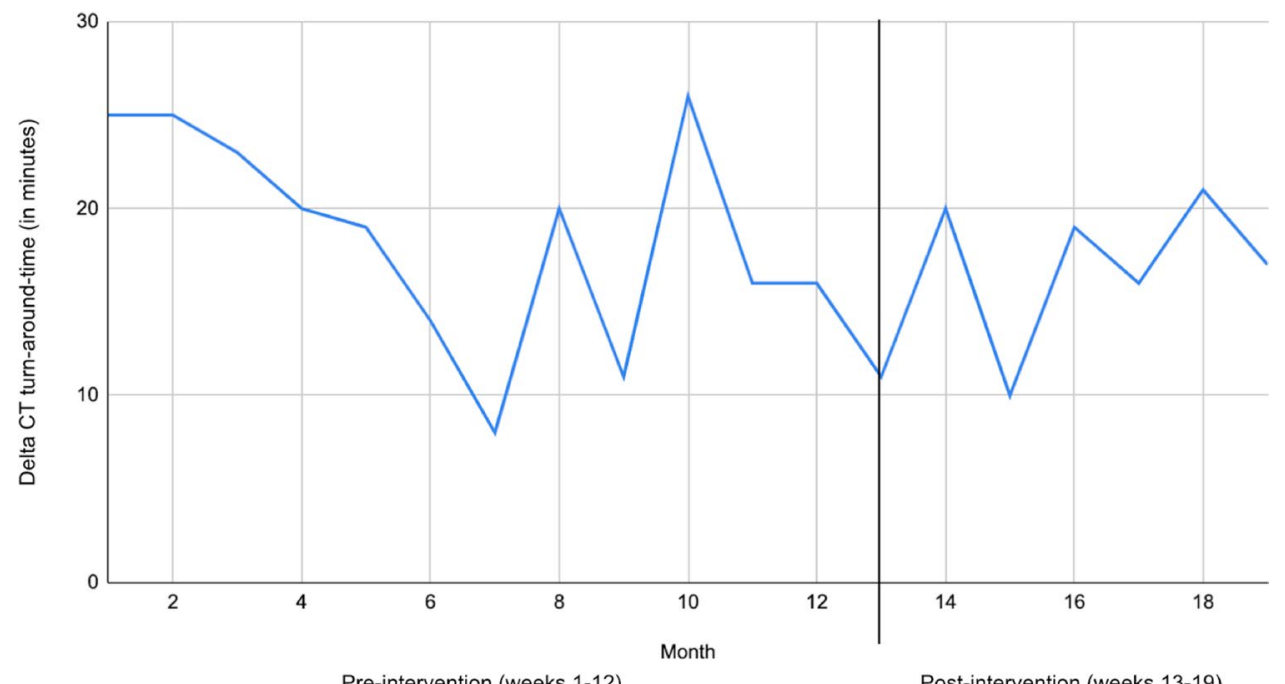

Pre-intervention (weeks 1-12)

Post-intervention (weeks 13-19)

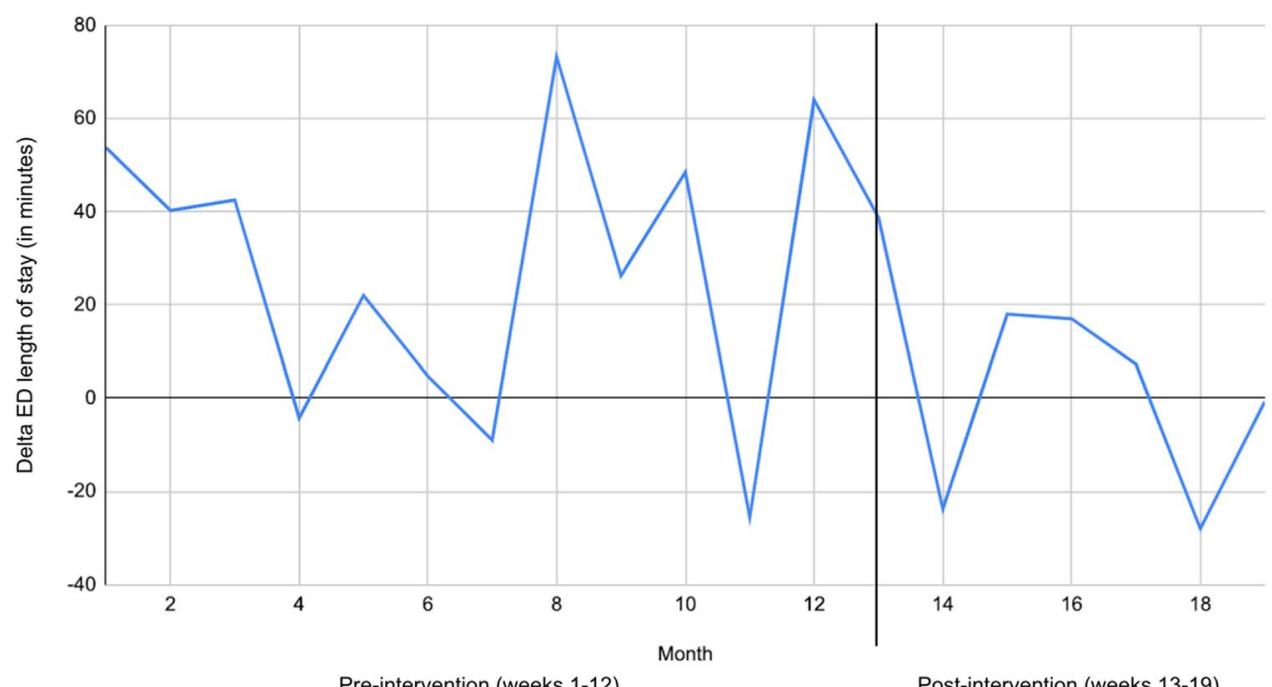

Pre-intervention (weeks 1-12)

Post-intervention (weeks 13-19)
Fig. 4 Run chart of the delta for ED length of stay for women of childbearing age compared to similarly aged men by month. Delta indicates the difference between the values for women of childbearing age and similarly age men. The vertical line represents the start of the intervention

Table 2 Pregnancy testing in all women of childbearing age and those who underwent CT chest and/or abdomen/pelvis over three time periods (pre-intervention, intensive intervention, and post-intervention) $\begin{array}{ll}\text { All women of childbear- } & \text { Women of childbearing age who } \\ \text { ing age } & \text { underwent CT chest and/or abdon }\end{array}$ pelvis

\begin{tabular}{llll}
\hline Pre-intervention & Total & 24,126 & 3252 \\
& Pregnancy tests ordered & $12,376(51.3 \%)$ & $2968(91.3 \%)$ \\
Intensive intervention & Pregnancy tests ordered from triage & $3271(26.4 \%)$ & 625 \\
& Total & 4631 & $575(92.0 \%)$ \\
& Pregnancy tests ordered & $2697(58.2 \%)^{*}$ & 1795 \\
Post-intervention & Pregnancy tests ordered from triage & $751(27.8 \%)$ & $2651(92.0 \%)$ \\
& Total & 14,229 & $7679(53.6 \%)^{*}$ \\
\hline
\end{tabular}

*Denotes statistically significant change from pre-intervention 
shows that our prior ad hoc process had room for improvement and demonstrates the potential impact of a streamlined screening process.

In regard to our primary aim, there are several possible reasons why our intervention was unsuccessful. First, our process measure may not have captured the full impact of our intervention. It is possible that some patients benefited from our intervention and had more timely pregnancy screening results prior to $\mathrm{CT}$ but were not included in our process measure (for instance, patients for which a urine sample was solicited but not ordered until rooming). Perhaps a more appropriate process measure would be the percentage of women of childbearing age who had a pregnancy test result documented prior to the $\mathrm{CT}$ technician arriving for transport. Second, patient factors may have affected our intervention's success, including resistance to pregnancy screening or request for provider evaluation prior to testing. Third, the ED Assistants underwent unanticipated staffing changes shortly after the start of our intervention, which reduced their availability and likely inhibited successful implementation. Additionally, the intervention focused on communication and training, which are less sustainable than interventions affecting organizational infrastructure and institutional processes [11]. Finally, our initiative involved rapid implementation on a large scale so it may have been difficult for staff to become well versed in the process. Because of these significant barriers to the success and sustainability of our intervention, we did not proceed with additional PDSA cycles after our initial quality improvement cycle.

\section{Comparison to previous studies}

The gender disparity we identified in radiology turn-aroundtime is consistent with results found in a prior study in which female stroke patients had significantly longer time to imaging compared to males [12]. We anticipated our intervention would lead to improved CT turn-around-time and ED length of stay in women of childbearing age and therefore an improvement in gender disparities. Towbin et al. found that improvements in radiology turn-around-time had a direct effect on overall ED flow by decreasing length of stay [13]. Additionally, there are several studies that investigated the association between ED pregnancy testing and efficiency of care in general for women [8, 14-16]. One abstract presented at the 2017 American College of Emergency Physicians Scientific Assembly demonstrated a significant reduction in length of stay for women undergoing imaging for abdominal pain with implementation of rapid serum pregnancy testing [14]. Our post-intervention results did not mirror the results of these studies.

In terms of ED pregnancy testing, our pre-intervention results are consistent with prior studies. One such study showed that $21 \%$ of adolescents presenting to the ED received pregnancy testing [17]. While our post-intervention data show an increase in the rate of testing that exceeds rates in other studies, there remains ample room for improvement.

\section{Limitations}

This project has several limitations. First, we did not compare emergency severity index, presenting complaints, or type of CT between groups, which could have represented confounding factors. Second, because this was a real-time quality improvement initiative, we were unable to control for seasonal changes in staffing and patient volumes between periods. We also considered that delays in CT turn-aroundtime for women of childbearing age were due to concurrent pelvic exams or simultaneous pelvic ultrasound testing. However, it is the culture of our ED to complete the full physical exam before ordering an imaging study and to order one imaging study at a time, so it is unlikely that a CT would be delayed due to an ongoing pelvic exam or ultrasound study. In addition, we did not obtain data for females over 50 years to evaluate for an etiology other than delays in pregnancy testing as a driver of gender disparities. Lastly, the project was conducted at a single hospital, and therefore its findings may not be generalizable to other institutions.

\section{Clinical implications}

This initiative confirms suspected gender disparities in CT turn-around-time and ED length of stay in our ED and calls attention to the importance of expedited pregnancy screening in this patient population. However, it is difficult to draw clinical implications from the intervention. Given our intervention was not successful, we cannot come to a conclusion regarding the etiology of the lack of improvement in CT turn-around-time and ED length of stay. It remains unclear whether the lack of improvement is due to factors other than pregnancy testing or if we simply did not improve the process enough to see change.

\section{Research implications}

Future research will need to incorporate more sustainable processes for pregnancy testing, such as implementing a rapid serum qualitative pregnancy test in triage. Once a better process for pregnancy testing is implemented, additional research can be conducted regarding its impact on CT turnaround-time and ED length of stay. 


\section{Conclusions}

Our project confirmed that gender disparities exist in our emergency department. There were longer CT turn-aroundtimes and ED lengths of stay for women of childbearing age compared to similarly aged men, highlighting an important area for improvement to promote equitable care. A quality improvement initiative with the aim to eliminate these disparities through protocolization of the pregnancy screening process in triage was not successful. A more sustainable intervention that expedites pregnancy screening, such as rapid serum pregnancy testing, is needed.

Acknowledgements The authors thank Sanjeev Malik MD, Michael Schmidt MD, Maribel Estrada RN, and James Adams MD for their mentorship and support of this study.

Author contributions DL, AF, JK, LA, VM, NS, and AF contributed to the design and implementation of the project. AF and DL were responsible for data collection and analysis. DL, AF, JK, LA, VM, NS, and $\mathrm{AF}$ contributed to writing and revisions of the manuscript.

Funding This research received no specific grant from any funding agency, commercial or not-for-profit sectors.

\section{Declarations}

Conflict of interest Abra Fant MD MS discloses the following financial remunerations, none of which are related to this quality improvement initiative or manuscript: AMA Redefining Residency Grant, speaking honoraria for the 2021 Southeast Michigan QI Summit, travel support for the AAMC Diagnostic Error Work Group In-Person Meeting, and leadership role as the ACGME Advisory Board Program Director for the Patient Safety Program.

\section{References}

1. Austin LM, Frush DP. Compendium of national guidelines for imaging the pregnant patient. Am J Roentgenol. 2011;197(4):W737-46. https://doi.org/10.2214/AJR.10.6351.

2. Abushouk AI, Sanei Taheri M, Pooransari P, et al. Pregnancy screening before diagnostic radiography in emergency department; an educational review. Emergency (Tehran). 2017;5(1):e60.

3. Herring A, Wilper A, Himmelstein DU, et al. Increasing length of stay among adult visits to US Emergency Departments, 20012005. Acad Emerg Med. 2009;16(7):609-16. https://doi.org/10. 1111/j.1553-2712.2009.00428.x.

4. Lauks J, Mramor B, Baumgartl K, et al. Medical team evaluation: effect on emergency department waiting time and length of stay. PLoS ONE. 2016;11(4):1-15. https://doi.org/10.1371/journ al.pone. 0154372 .

5. Bernstein SL, Aronsky D, Duseja R, et al. The effect of emergency department crowding on clinically oriented outcomes. Acad Emerg Med. 2009;16(1):1-10. https://doi.org/10.1111/j. 1553-2712.2008.00295.x.

6. Cournane S, Conway R, Creagh D, et al. Radiology imaging delays as independent predictors of length of stay for emergency medical admissions. Clin Radiol. 2016;71(9):912-8. https://doi. org/10.1016/j.crad.2016.03.023.

7. Quidel QuickVue hCG Urine Test Package Insert. 2021. Available from: https://www.quidel.com/immunoassays/rapid-pregnancytests/quickvue-hcg-urine-test. Accessed 24 July 2021

8. Lazarenko G, Dobson C, Enokson R, et al. Accuracy and speed of urine pregnancy tests done in the emergency department: a prospective study. CJEM. 2001;3(4):292-5. https://doi.org/10.1017/ S1481803500005790.

9. American College of Radiology. ACR-SPR Practice parameter for imaging pregnant of potentially pregnant adolescents and women with ionizing radiation. 2018. Available from: https://www.acr. org/Clinical-Resources/Radiology-Safety/Radiation-Safety. Access 31 July 2021

10. Ogrinc G, Davies L, Goodman D. SQUIRE 2.0 (Standards for Quality Improvement Reporting Excellence): revised publication guidelines from a detailed consensus process. BMJ Qual Saf. 2016;25:986-92. https://doi.org/10.1136/bmjqs-2015-004411.

11. Dixon-Woods M, McNicol S, Martin G. Ten challenges in improving quality in healthcare: lessons from the Health Foundation's programme evaluations and relevant literature. BMJ Qual Saf. 2012;21(10):876-84. https://doi.org/10.1136/bmjqs-2011-000760.

12. Park SJ, Shin SD, Ro YS, et al. Gender differences in emergency stroke care and hospital outcome in acute ischemic stroke: a multicenter observational study. Am J Emerg Med. 2013;31(1):178-84. https://doi.org/10.1016/j.ajem.2012.07.004.

13. Towbin AJ, Iyer SB, Brown J, et al. Practice policy and quality initiatives: decreasing variability in turnaround time for radiographic studies from the emergency department. Radiographics. 2013;33(2):361-71. https://doi.org/10.1148/rg.332125738.

14. Fuentes R, Naeem TH, Lui H, et al. 16 Serum vs urine pregnancy test: the effect on emergency department disposition times in females with abdominal pain requiring radiographic study. Ann Emerg Med. 2017;70(4):S7. https://doi.org/10.1016/j.annem ergmed.2017.07.040.

15. Fromm C, Likourezos A, Haines L, et al. Substituting whole blood for urine in a bedside pregnancy test. J Emerg Med. 2012;43(3):478-82. https://doi.org/10.1016/j.jemermed.2011. 05.028 .

16. Singer A, Taylor M, Leblanc D, et al. Early point-of-care testing at triage reduces care time in stable adult emergency department patients. J Emerg Med. 2018;55(2):172-8. https://doi.org/10. 1016/j.jemermed.2018.04.061.

17. Goyal M, Hersh A, Luan X, et al. Frequency of pregnancy testing among adolescent emergency department visits. Acad Emerg Med. 2013;20(8):816-21. https://doi.org/10.1111/acem.12186. 